\title{
AN APPLICATION OF SET THEORY TO THE TORSION PRODUCT OF ABELIAN GROUPS
}

\author{
PATRICK KEEF
}

(Communicated by Ron Solomon)

\begin{abstract}
The following problem of Fuchs is considered: relate the abelian groups $A$ and $B$ assuming $\operatorname{Tor}(A, G) \cong \operatorname{Tor}(B, G)$ for all reduced abelian groups $G$. A complete characterization is obtained in any set-theoretic universe in which $E(\kappa)$ is valid for a proper class of regular cardinals $\kappa$.
\end{abstract}

In the well-known list of 100 questions posed in [3], problem 50 asks us to relate the abelian groups $A$ and $B$ assuming $\operatorname{Tor}(A, G) \cong \operatorname{Tor}(B, G)$ for all reduced abelian groups $G$. There is clearly no loss of generality in assuming $A$, $B$, and $G$ are $p$-groups for some fixed prime $p$, so hereafter the term "group" will mean "abelian p-group." Hill showed in [4] that $A$ and $B$ share many properties but left unresolved whether they must necessarily be isomorphic. Cutler showed in [1] that this need not be the case. Specifically, groups $A$ and $C$ were found such that $C$ is a direct sum of cyclics, $\operatorname{Tor}(A, G) \cong \operatorname{Tor}(A \oplus C, G)$ for all reduced $G$, but $A \neq A \oplus C$. Is this the only way to construct such examples? In other words, if $A$ and $B$ satisfy problem 50, do they necessarily differ by summands which are direct sums of cyclics?

Denote the Ulm function of the group $Y$ by $f_{Y}$. We will say $A$ and $B$ are $T$-equivalent if

(a) $f_{A}(n)=f_{B}(n)$ for all $n<\omega$, and

(b) for some direct sums of cyclics $X$ and $Y, A \oplus X \cong B \oplus Y$.

We show that if $A$ and $B$ are $T$-equivalent, then they satisfy problem 50 (Theorem 2). Conversely, if $A$ and $B$ satisfy problem 50 and there is a regular cardinal $\kappa$ greater than the ranks of $A$ and $B$ such the $E(\kappa)$ is valid, we show that $A$ and $B$ must be $T$-equivalent (Theorem 4; we review these set-theoretic terms later). In particular, we have a solution for problem 50 in any set-theoretic universe in which $E(\kappa)$ is valid for a proper class of regular cardinals. This will be true, for example, in the constructible universe.

We first review the treatment of Ulm invariants contained in [5]. If $Z$ is a valuated group and $\alpha$ is an ordinal, let $k_{Z}(\alpha)$ be the kernel of the map $Z(\alpha) / Z(\alpha+1) \stackrel{\times p}{\rightarrow} Z(\alpha+1) / Z(\alpha+2)$, so $f_{Z}(\alpha)$ is the dimension of $k_{Z}(\alpha)$ as a

Received by the editors November 11, 1991.

1991 Mathematics Subject Classification. Primary 20K10, 20 K40.

Key words and phrases. Abelian p-group, torsion product.

The author was supported by NSF (RUI) grant DMS-9000129. 
$\mathbb{Z}_{p}$-vector space. If $Z$ is a subgroup of $Y$ and, for $z \in Z, v(z)=\mathrm{ht}_{Y}(z)$, then the $\alpha$ th relative Ulm invariant, $f_{Y, Z}(\alpha)$, is the dimension of the cokernel of the induced injection $k_{Z}(\alpha) \rightarrow k_{Y}(\alpha)$. It follows that $f_{Y}(\alpha)=f_{Y, Z}(\alpha)+f_{Z}(\alpha)$. We apply this in

Lemma 1. Suppose $A$ and $B$ are T-equivalent. Then there are direct sums of cyclics $X$ and $Y$ such that

(a) $f_{A \oplus X}(n)=f_{A}(n)=f_{B}(n)=f_{B \oplus Y}(n)$ for all $n<\omega$, and

(b) $A \oplus X \cong B \oplus Y$.

Proof. For some direct sums of cyclics $X^{\prime}$ and $Y^{\prime}, A \oplus X^{\prime} \cong B \oplus Y^{\prime}$; we identify these and call the result $H$. Let $G=A \cap B$. Note that for all $g \in G$, $\mathrm{ht}_{A}(g)=\mathrm{ht}_{H}(g)=\mathrm{ht}_{B}(g)$, so $\mathrm{id}_{G}: G \rightarrow G$ preserves heights computed in $A$ and $B$, respectively. Since $A / G$ embeds in $Y^{\prime}$, it is a direct sum of cyclics; similarly, $B / G$ is a direct sum of cyclics. Let $f=f_{A}=f_{B}$. If $G$ is given the valuation induced by the height function on $H$, then for every $n<\omega$,

$$
f_{A, G}(n)+f_{G}(n)=f(n)=f_{B, G}(n)+f_{G}(n) .
$$

Let $X$ be a direct sum of cyclics such that $f_{X}(n)+f_{A, G}(n)=f_{B, G}(n)$ whenever $f_{A, G}(n)<f_{B, G}(n)$, and $f_{X}(n)=0$ otherwise. Similarly, let $Y$ be a direct sum of cyclics such that $f_{Y}(n)+f_{B, G}(n)=f_{A, G}(n)$ whenever $f_{B, G}(n)<f_{A, G}(n)$, and $f_{Y}(n)=0$ otherwise. It follows that for all $n<\omega$,

$$
f(n)+f_{X}(n)=f(n)=f(n)+f_{Y}(n)
$$

so that $(a)$ is valid. Further,

$$
f_{A \oplus X, G \oplus 0}(n)=f_{A, G}(n)+f_{X}(n)=f_{B, G}(n)+f_{Y}(n)=f_{B \oplus Y, G \oplus 0}(n) .
$$

So by [3, 83.4], $\operatorname{id}_{G \oplus 0}$ extends to an isomorphism $A \oplus X \cong B \oplus Y$, as required.

Theorem 2. If $A$ and $B$ are $T$-equivalent, then for every reduced group $G$, $\operatorname{Tor}(A, G) \cong \operatorname{Tor}(B, G)$.

Proof. Let $X$ and $Y$ be as in Lemma 1. We will be done if we can show $\operatorname{Tor}(A, G) \cong \operatorname{Tor}(A \oplus X, G)$ for all reduced $G$, since similar isomorphisms will be true for $B$ and $A \oplus X \cong B \oplus Y$. It is readily checked that our hypothesis guarantees that for every $n<\omega, r\left(p^{n} A\right)=r\left(p^{n}(A \oplus X)\right)$. By [3, 64.4] for any groups $M$ and $N$ and ordinal $\alpha$ there is an equation

$$
f_{\operatorname{Tor}(M, N)}(\alpha)=f_{M}(\alpha) f_{N}(\alpha)+f_{M}(\alpha) r\left(p^{\alpha+1} N\right)+r\left(p^{\alpha+1} M\right) f_{N}(\alpha) .
$$

If $\operatorname{Tor}(A, G)$ is a direct sum of cyclic groups then so is $\operatorname{Tor}(A \oplus X, G)$, and it follows from $(*)$ that $\operatorname{Tor}(A, G) \cong \operatorname{Tor}(A \oplus X, G)$. In particular, if either $A$ or $G$ is bounded, the isomorphism follows. Suppose both $A$ and $G$ are unbounded. If $Z$ is isomorphic to a basic subgroup of $\operatorname{Tor}(A, G)$, it follows from $(*)$ that $Z \cong Z \oplus Z$. By [6, 1.7] $\operatorname{Tor}(A, G) \cong \operatorname{Tor}(A, G) \oplus Z$. Since $\operatorname{Tor}(X, G)$ is a direct sum of cyclics and by $(*), f_{\operatorname{Tor}(X, G)}(n) \leq f_{Z}(n)$ for all $n<\omega$, it follows that $Z \cong Z \oplus \operatorname{Tor}(X, G)$. Therefore,

$$
\begin{aligned}
\operatorname{Tor}(A, G) & \cong \operatorname{Tor}(A, G) \oplus Z \cong \operatorname{Tor}(A, G) \oplus Z \oplus \operatorname{Tor}(X, G) \\
& \cong \operatorname{Tor}(A, G) \oplus \operatorname{Tor}(X, G) \cong \operatorname{Tor}(A \oplus X, G),
\end{aligned}
$$

as required. 
We now review some notions from set theory. Suppose $\alpha$ is a limit ordinal. A subset $C \subseteq \alpha$ is a $c u b$ if it is closed (in the order topology) and unbounded. A subset $S \subseteq \alpha$ is stationary if for every cub $C \subseteq \alpha, S \cap C \neq \varnothing$. The cofinality of $\alpha$ is the minimal cardinality of its unbounded subsets. A cardinal $\kappa$ is regular if it has cofinality $\kappa$. If $\kappa$ is a regular cardinal, then $E(\kappa)$ is the following statement:

There is a stationary subset $S \subseteq \kappa$ such that (1) every $\alpha \in S$ is a limit ordinal of countable cofinality, and (2) for every limit ordinal $\alpha<\kappa, \alpha \cap S$ is not a stationary subset of $\alpha$.

If $G$ is a group of cardinals, then a collection of subgroups $\left\{W_{j}\right\}_{j<\alpha}$ whose union is $G$ is a $\kappa$-filtration if for every $j<\alpha, W_{j}=\bigcup_{i<j} W_{i+1}$ and $\left|W_{j}\right|<|G|$.

Theorem 3. Suppose $\kappa$ is a regular cardinal for which $E(\kappa)$ is valid and $S \subseteq \kappa$ is as in the definition of $E(\kappa)$. Then there is a group $G$ of cardinality $\kappa$ with a $\kappa$-filtration $\left\{W_{j}\right\}_{j<\kappa}$ consisting of pure subgroups, such that whenever $j<l<\kappa$ and $j \in S, W_{l} / W_{j} \cong \mathbb{Z}_{p^{\infty}} \oplus M$, where $M$ is a direct sum of cyclics.

Proof. For every $i<\kappa$ and $m<\omega$, let $C_{m}^{i} \cong \mathbb{Z}_{p^{m}}$ and $B^{i}=\bigoplus_{m<\omega} C_{m}^{i}$. For a moment, fix $j \in S$ and let $x_{1}, x_{2}, x_{3}, \ldots$ be a strictly increasing sequence of isolated ordinals with limit $j$. Let $\bigoplus_{m<\omega} C_{m}^{x_{m}} \subseteq Z_{j} \subseteq \prod_{m<\omega} C_{m}^{x_{m}}$ satisfy $Z_{j} / \bigoplus_{m<\omega} C_{m}^{x_{m}} \cong \mathbb{Z}_{p^{\infty}}$. Let $G \subseteq \prod_{i<\kappa} B^{i}$ be generated by $\bigoplus_{i<\kappa} B^{i}$ and the $Z_{j}$ 's for $j \in S$. If $j<\kappa$, let $G_{j}=G \cap \prod_{i<j} B^{i}$ and $G^{j}=G \cap \prod_{j \leq i<\kappa} B^{i}$. Observe that if $j<\kappa$ and $\pi_{j}: \prod_{i<\kappa} B^{i} \rightarrow \prod_{i<j} B^{i}$ is the obvious projection, then whenever $l>j$ is in $S$, we have $\pi_{j}\left(Z_{l}\right) \subseteq \bigoplus_{i<j} B^{i}$. Therefore, $\pi_{j}(G) \subseteq$ $G$, so that $G \cong G_{j} \oplus G^{j}$. In addition $G_{j}$ will be generated by $\bigoplus_{i<j} B^{i}$ and the $Z_{i}$ for $i \leq j$ with $i \in S$. In particular, $\left|G_{j}\right|=|j| \cdot \omega_{0}$. Finally, let $W_{j}=\bigcup_{i<j} G_{i}$. Clearly these form a $\kappa$-filtration of $G$ by pure subgroups.

We claim that $G_{j}$ is a direct sum of cyclics for all $j<\kappa$. We prove this by induction. Since $G_{j+1} \cong G_{j} \oplus B^{j}$, the interesting case is where $j$ is a limit ordinal. Let $g: \mu \rightarrow j-S$ be a strictly increasing function whose image is a cub. For each $\beta<\mu, G_{g(\beta)}$ is a direct sum of cyclics which is a summand of $G$, hence, of $G_{g(\beta+1)}$. Note that if $\sigma<\mu$ is a limit, then $g(\sigma)$ is not in $S$, so that $G_{g(\sigma)}=W_{g(\sigma)}$. Therefore, $W_{j} \cong \bigoplus_{\beta<\mu} G_{g(\beta+1)} / G_{g(\beta)}$ is a direct sum of cyclics. Note that if $j \notin S$, then $G_{j}=W_{j}$ and the claim follows, and if $j \in S$, then $G_{j}$ is generated by $W_{j}$, and $Z_{j}$, hence, $G_{j} / W_{j} \cong \mathbb{Z}_{p^{\infty}}$. There is a decomposition $W_{j}=K \oplus L$, where $L$ is a countable subgroup containing $W_{j} \cap Z_{j}$. Clearly $K$ is a direct sum of cyclics, and since $L+Z_{j}$ is countable, it is a direct sum of cyclics, also. Therefore $G_{j}=K \oplus\left(L+Z_{j}\right)$ is a direct sum of cyclics, as required.

Note that from the last paragraph, if $j \in S$ and $j<l<\kappa$, then

$$
\mathbb{Z}_{p^{\infty}} \cong G_{j} / W_{j} \subseteq W_{l} / W_{j} \subseteq G_{l} / W_{j},
$$

and therefore $W_{l} / W_{j} \cong \mathbb{Z}_{p^{\infty}} \oplus M$, where $M \subseteq G_{l} / G_{j}$ is a direct sum of cyclics.

Let $\lambda$ be the supremum of the values of $\kappa$ for which $E(\kappa)$ is valid. So $\lambda$ is either a cardinal or the symbol $\infty$ if $E(\kappa)$ is valid for a proper class of regular cardinals.

Observe that if $A[p] \cong \operatorname{Tor}\left(A, \mathbb{Z}_{p}\right) \cong \operatorname{Tor}\left(B, \mathbb{Z}_{p}\right) \cong B[p]$, then $A$ and $B$ have the same rank. 
Theorem 4. Suppose $\operatorname{Tor}(A, G) \cong \operatorname{Tor}(B, G)$ for every reduced $G$ and the rank of $A$ and $B$ is less than $\lambda$. Then $A$ and $B$ are T-equivalent.

Proof. By [4, Theorem 1] $A$ and $B$ have the same Ulm invariants. Let $\kappa$ be a regular cardinal greater than the ranks of $A$ and $B$ for which $E(\kappa)$ holds and let $S \subseteq \kappa, G$, and $\left\{W_{j}\right\}_{j<\kappa}$ be as in Theorem 3. Suppose $g$ : $\operatorname{Tor}(A, G) \rightarrow$ $\operatorname{Tor}(B, G)$ is an isomorphism. Note that $\left\{\operatorname{Tor}\left(A, W_{j}\right)\right\}_{j<\kappa},\left\{\operatorname{Tor}\left(B, W_{j}\right)\right\}_{j<\kappa}$ are $\kappa$-filtrations of $\operatorname{Tor}(A, G)$, $\operatorname{Tor}(B, G)$, respectively. By a simple back-andforth argument, there is a cub $C \subseteq \kappa$ such that for all $j \in C, g\left(\operatorname{Tor}\left(A, W_{j}\right)\right)=$ $\operatorname{Tor}\left(B, W_{j}\right)$. Since $S$ is stationary, there is a $j \in S \cap C$. Let $l>j$ with $l \in C$. Since $W_{j}$ is a pure subgroup of $W_{l}$ and Tor is exact on pure sequences [3, 63.2], it follows that there is a commutative diagram

$$
\begin{aligned}
& 0 \rightarrow \operatorname{Tor}\left(A, W_{j}\right) \rightarrow \operatorname{Tor}\left(A, W_{l}\right) \rightarrow \operatorname{Tor}\left(A, W_{l} / W_{j}\right) \rightarrow 0 \\
& \downarrow \cong \quad \downarrow \cong \quad \downarrow \cong \\
& 0 \rightarrow \operatorname{Tor}\left(B, W_{j}\right) \rightarrow \operatorname{Tor}\left(B, W_{l}\right) \rightarrow \operatorname{Tor}\left(B, W_{l} / W_{j}\right) \rightarrow 0
\end{aligned}
$$

If $W_{l} / W_{j} \cong \mathbb{Z}_{p^{\infty}} \oplus M$, then $X=\operatorname{Tor}(A, M)$ and $Y=\operatorname{Tor}(B, M)$ are direct sums of cyclics and

$$
A \oplus X \cong \operatorname{Tor}\left(A, W_{l} / W_{j}\right) \cong \operatorname{Tor}\left(B, W_{l} / W_{j}\right) \cong B \oplus Y,
$$

which proves the result.

Corollary 5. If $\lambda=\infty$, then $\operatorname{Tor}(A, G) \cong \operatorname{Tor}(B, G)$ for all reduced $G$ iff $A$ and $B$ are T-equivalent.

We conclude with a discussion of the condition $\lambda=\infty$. As noted above, this is true in $V=L$. More generally, if there is a proper class of successor cardinals in $L$ which are also cardinals in $V$, then $\lambda=\infty$ [2, VI, 3.6]. This holds, for example, when $0^{\#}$ does not exist [2, VI, 3.16]. The condition $\lambda<\infty$ is related to the existence of large cardinals of certain types. For example, if $\lambda<\infty$, then it is consistent that the measurable cardinals form a proper class (this follows from [2, VI, 3.17], since there are arbitrarily large singular cardinals). Conversely, if there is a $L_{\omega_{1} \omega}$-compact cardinal, then we can conclude that $\lambda<\infty$ [2, VI, 3.18]. Note that if $\lambda<\infty$, it is still possible that any two groups satisfying problem 50 are $T$-equivalent, but some other technique will have to be employed to prove this.

\section{REFERENCES}

1. D. Cutler, Abelian p-groups $A$ and $B$ such that $\operatorname{Tor}(A, G) \cong \operatorname{Tor}(B, G), G$ reduced, Proc. Amer. Math. Soc. 91 (1984), 12-14.

2. P. Eklof and A. Mekler, Almost free modules, set-theoretic methods, North-Holland, Amsterdam, 1990.

3. L. Fuchs, Infinite abelian groups, Vols. 1 and 2, Academic Press, New York, 1970 and 1973.

4. P. Hill, Two problems of Fuchs concerning tor and hom, J. Algebra 19 (1971), 379-383.

5. R. Hunter, F. Richman, and E. Walker, Warfield modules, Lecture Notes in Math., vol. 616, Springer-Verlag, Berlin and New York, 1977, pp. 87-123.

6. P. Keef, Some isomorphisms of abelian groups involving the Tor functor, Proc. Amer. Math. Soc. 110 (1990), 27-37.

Department of Mathematics, Whitman College, Walla Walla, Washington 99362 E-mail address: KEEF@WHITMAN.BITNET 